PROCEEDINGS OF THE

AMERICAN MATHEMATICAL SOCIETY

Volume 137, Number 2, February 2009, Pages 711-721

S 0002-9939(08)09546-4

Article electronically published on September 4, 2008

\title{
SPACELIKE HYPERSURFACES WITH CONSTANT MEAN CURVATURE IN THE STEADY STATE SPACE
}

\author{
ALMA L. ALBUJER AND LUIS J. ALÍAS \\ (Communicated by Richard A. Wentworth)
}

\begin{abstract}
We consider complete spacelike hypersurfaces with constant mean curvature in the open region of de Sitter space known as the steady state space. We prove that if the hypersurface is bounded away from the infinity of the ambient space, then the mean curvature must be $H=1$. Moreover, in the 2-dimensional case we obtain that the only complete spacelike surfaces with constant mean curvature which are bounded away from the infinity are the totally umbilical flat surfaces. We also derive some other consequences for hypersurfaces which are bounded away from the future infinity. Finally, using an isometrically equivalent model for the steady state space, we extend our results to a wider family of spacetimes.
\end{abstract}

\section{The STEAdy STATE SPACE}

Let $\mathbb{R}_{1}^{n+2}$ be the $(n+2)$-dimensional Lorentz-Minkowski space, that is, the real vector space $\mathbb{R}^{n+2}$ endowed with the Lorentzian metric

$$
\langle,\rangle=-\left(d x_{0}\right)^{2}+\left(d x_{1}\right)^{2}+\ldots+\left(d x_{n+1}\right)^{2}
$$

where $\left(x_{0}, \ldots, x_{n+1}\right)$ are the canonical coordinates of $\mathbb{R}^{n+2}$. As it is well known, the hyperquadric

$$
\mathbb{S}_{1}^{n+1}=\left\{x \in \mathbb{R}_{1}^{n+2}:\langle x, x\rangle=1\right\}
$$

consisting of all unit spacelike vectors in $\mathbb{R}_{1}^{n+2}$ endowed with the induced metric from $\mathbb{R}_{1}^{n+2}$ is the de Sitter space. The de Sitter space is a complete simply connected $(n+1)$-dimensional Lorentzian manifold with constant sectional curvature one. Therefore, $\mathbb{S}_{1}^{n+1}$ can be seen, in Lorentzian geometry, as the equivalent to the Euclidean sphere.

Received by the editors May 31, 2007, and, in revised form, February 5, 2008.

2000 Mathematics Subject Classification. Primary 53C42, 53C50.

Key words and phrases. de Sitter space, steady state space, spacelike hypersurface, mean curvature, parabolicity.

The first author was supported by FPU Grant AP2004-4087 from Secretaría de Estado de Universidades e Investigación, MEC Spain.

This research is a result of the activity developed within the framework of the Programme in Support of Excellence Groups of the Región de Murcia, Spain, by Fundación Séneca, Regional Agency for Science and Technology (Regional Plan for Science and Technology 2007-2010).

This research was partially supported by MEC project MTM2007-64504 and Fundación Séneca project 04540/GERM/06, Spain.

(C)2008 American Mathematical Society Reverts to public domain 28 years from publication 
Now take a non-zero null vector $a \in \mathbb{R}_{1}^{n+2}$ past-pointing, that is, $\langle a, a\rangle=0$ and $\left\langle a, e_{0}\right\rangle>0$ where $e_{0}=(1,0, \ldots, 0)$, and consider the following open region of the de Sitter space:

$$
\mathcal{H}^{n+1}=\left\{x \in \mathbb{S}_{1}^{n+1}:\langle x, a\rangle>0\right\} .
$$

The open region $\mathcal{H}^{n+1}$ forms the spacetime for the steady state model of the universe proposed by Bondi and Gold [4] and Hoyle [8] when one is looking for a model of the universe which looks the same not only at all points and in all directions (that is, spatially isotropic and homogeneous), but at all times (cf. [14, Section 14.8] and [7. Section 5.2]). The steady state space is a non-complete manifold, being only half of the de Sitter space and having as its boundary the null hypersurface

$$
L_{0}=\left\{x \in \mathbb{S}_{1}^{n+1}:\langle x, a\rangle=0\right\} .
$$

The null hypersurface $L_{0}$ represents the past infinity of $\mathcal{H}^{n+1}$, usually denoted by $\mathcal{J}^{-}$, and the limit boundary

$$
L_{\infty}=\left\{x \in \mathbb{S}_{1}^{n+1}:\langle x, a\rangle=\infty\right\}
$$

represents its future infinity, usually denoted by $\mathcal{J}^{+}$.

\section{SPACElike hypersurfaces in the Steady State SPACE}

A smooth immersion $\psi: \Sigma^{n} \rightarrow \mathcal{H}^{n+1}$ of an $n$-dimensional connected manifold $\Sigma^{n}$ is said to be a spacelike hypersurface if $\psi$ induces a Riemannian metric on $\Sigma$, which as usual is also denoted by $\langle$,$\rangle . In that case, there exists a unique unitary$ timelike normal field $N$ globally defined on $\Sigma$ which is future-directed. Throughout this paper we will refer to $N$ as the future-pointing Gauss map of $\Sigma$. The mean curvature function of a spacelike hypersurface $\Sigma$ is defined as

$$
H=-\frac{1}{n} \operatorname{tr}(A)
$$

where $A$ stands for the shape operator (or second fundamental form) of $\Sigma$ with respect to its future-pointing Gauss map $N$. The choice of the sign "_" in our definition of $H$ is motivated by the fact that in that case the mean curvature vector is given by $\vec{H}=H N$. Therefore, $H(p)>0$ at a point $p \in \Sigma$ if and only if $\vec{H}(p)$ is future-directed.

The steady state space has a special property: it admits a foliation by means of totally umbilical spacelike hypersurfaces

$$
L_{\tau}=\left\{x \in \mathcal{H}^{n+1}:\langle x, a\rangle=\tau\right\}, \quad \tau>0,
$$

having constant mean curvature $H=1$ with respect to their future-pointing Gauss map

$$
N_{\tau}(x)=x-\frac{1}{\tau} a .
$$

It is worth pointing out that each $L_{\tau}$ is isometric to the Euclidean space $\mathbb{R}^{n}$. Actually, if we identify $\mathbb{R}^{n}$ with the orthogonal complement in $\mathbb{R}_{1}^{n+2}$ of the Lorentzian plane spanned by $a$ and $e_{0}$, then it is easy to see that the map $\phi_{\tau}: L_{\tau} \rightarrow \mathbb{R}^{n}$ given by

$$
\phi_{\tau}(x)=x-\frac{\tau+\left\langle a, e_{0}\right\rangle\left\langle x, e_{0}\right\rangle}{\left\langle a, e_{0}\right\rangle^{2}} a-\frac{\tau}{\left\langle a, e_{0}\right\rangle} e_{0}
$$

defines an isometry between $L_{\tau}$ and $\mathbb{R}^{n}$. 
In this context, we will say that a spacelike hypersurface $\Sigma$ in $\mathcal{H}^{n+1}$ is bounded away from the future infinity if there exists $\bar{\tau}>0$ such that

$$
\psi(\Sigma) \subset\left\{x \in \mathcal{H}^{n+1}:\langle x, a\rangle \leq \bar{\tau}\right\},
$$

and we will say that it is bounded away from the past infinity if there exists $\underline{\tau}>0$ such that

$$
\psi(\Sigma) \subset\left\{x \in \mathcal{H}^{n+1}:\langle x, a\rangle \geq \underline{\tau}\right\} .
$$

We will say that $\Sigma$ is bounded away from the infinity if it is bounded away both from the past and from the future infinity. In other words, $\Sigma$ is bounded away from the infinity if there exist $0<\underline{\tau}<\bar{\tau}$ such that $\psi(\Sigma)$ is contained in the slab bounded by $L_{\underline{\tau}}$ and $L_{\bar{\tau}}$.

Lemma 1. Let $\psi: \Sigma^{n} \rightarrow \mathcal{H}^{n+1}$ be a complete spacelike hypersurface. If $\Sigma$ is bounded away from the future infinity of $\mathcal{H}^{n+1}$, then $\Sigma$ is diffeomorphic to $\mathbb{R}^{n}$. In particular, there is no compact (without boundary) spacelike hypersurface in $\mathcal{H}^{n+1}$.

Proof. We will see that $\Sigma$ is diffeomorphic to $\mathbb{R}^{n}$ by showing that its orthogonal geodesic projection onto $L_{1}$ is a diffeomorphism. Observe that the steady state spacetime $\mathcal{H}^{n+1}$ can be globally parametrized by means of the diffeomorphism $\Phi: \mathbb{R} \times L_{1} \rightarrow \mathcal{H}^{n+1}$ given by $\Phi(t, q)=\gamma_{q}(t)$, where

$$
\gamma_{q}(t)=e^{t} q-\sinh t a
$$

denotes the (future pointing) unitary geodesic orthogonal to $L_{1}$ through the point $q \in L_{1}$. Then, the orthogonal geodesic projection of $\mathcal{H}^{n+1}$ onto $L_{1}$ assigns to each $x \in \mathcal{H}^{n+1}$ the point $\Psi(x) \in L_{1}$ such that $x=\Phi(t, \Psi(x))$ for a certain $t$, and it is given by

$$
\Psi(x)=\frac{1}{\langle x, a\rangle} x+\frac{1}{2}\left(1-\frac{1}{\langle x, a\rangle^{2}}\right) a, \quad x \in \mathcal{H}^{n+1} .
$$

Let $\psi: \Sigma^{n} \rightarrow \mathcal{H}^{n+1}$ be a spacelike hypersurface. The orthogonal geodesic projection of $\Sigma$ onto $L_{1}$ is the composition map $\Pi=\Psi \circ \psi: \Sigma \rightarrow L_{1}$ written as

$$
\Pi=\frac{1}{\langle\psi, a\rangle} \psi+\frac{1}{2}\left(1-\frac{1}{\langle\psi, a\rangle^{2}}\right) a .
$$

A straightforward computation shows that

$$
d \Pi_{p}(v)=\frac{1}{\langle\psi(p), a\rangle} d \psi_{p}(v)-\frac{\left\langle d \psi_{p}(v), a\right\rangle}{\langle\psi(p), a\rangle^{2}} \psi(p)+\frac{\left\langle d \psi_{p}(v), a\right\rangle}{\langle\psi(p), a\rangle^{3}} a
$$

for every $p \in \Sigma$ and every tangent vector $v \in T_{p} \Sigma$. Therefore,

$$
\begin{aligned}
\left\langle d \Pi_{p}(v), d \Pi_{p}(v)\right\rangle_{\mathrm{o}} & =\frac{1}{\langle\psi(p), a\rangle^{2}}\left\langle d \psi_{p}(v), d \psi_{p}(v)\right\rangle+\frac{\left\langle d \psi_{p}(v), a\right\rangle^{2}}{\langle\psi(p), a\rangle^{4}} \\
& \geq \frac{1}{\langle\psi(p), a\rangle^{2}}\left\langle d \psi_{p}(v), d \psi_{p}(v)\right\rangle,
\end{aligned}
$$

that is,

$$
\Pi^{*}\left(\langle,\rangle_{\mathrm{o}}\right) \geq \frac{1}{\langle\psi, a\rangle^{2}}\langle,\rangle
$$


where $\langle,\rangle_{\mathrm{o}}$ denotes the flat Euclidean metric on $L_{1}$ and $\langle$,$\rangle denotes the Riemannian$ metric on $\Sigma$. Since we are assuming that $\Sigma$ is bounded away from the future infinity of $\mathcal{H}^{n+1}$, it follows from here that

$$
\Pi^{*}\left(\langle,\rangle_{\mathrm{o}}\right) \geq \frac{1}{\bar{\tau}^{2}}\langle,\rangle
$$

for certain positive $\bar{\tau}$.

From (11) we get that $\Pi$ is a local diffeomorphism. Since $\langle$,$\rangle is a complete$ Riemannian metric on $\Sigma$, the same holds for the homothetic metric $\widetilde{\langle,\rangle}=\left(1 / \bar{\tau}^{2}\right)\langle$,$\rangle .$ Then, (2) means that the map

$$
\Pi:(\Sigma, \widetilde{\langle,\rangle}) \rightarrow\left(L_{1},\langle,\rangle_{\mathrm{o}}\right)
$$

increases the distance, and hence $\Pi$ is a covering map by 10, Chapter VIII, Lemma 8.1]. But $L_{1}$ being simply connected means that $\Pi$ is in fact a global diffeomorphism between $\Sigma$ and $L_{1}$.

\section{Spacelike hypersurfaces With CONStant mean CURVAture}

For a spacelike hypersurface $\psi: \Sigma^{n} \rightarrow \mathcal{H}^{n+1}$, consider the function $u \in \mathcal{C}^{\infty}(\Sigma)$ defined as $u(p)=\langle\psi(p), a\rangle, p \in \Sigma$. Observe that the gradient of $u$ in $\Sigma$ is

$$
\nabla u=a^{\top}
$$

where $a^{\top}$ denotes the tangential component of $a$ along $\Sigma$, that is,

$$
a=a^{\top}-\langle N, a\rangle N+\langle\psi, a\rangle \psi=\nabla u-\langle N, a\rangle N+\langle\psi, a\rangle \psi .
$$

Using Gauss and Weingarten formulae, we easily obtain

$$
\nabla_{X} \nabla u=-\langle N, a\rangle A X-u X
$$

for every $X \in T \Sigma$. Therefore, the Laplacian of the function $u$ on $\Sigma$ is given by

$$
\Delta u=n H\langle N, a\rangle-n u .
$$

From (3), it is also easy to see that

$$
\|\nabla u\|^{2}=\langle N, a\rangle^{2}-u^{2}
$$

where $\|\cdot\|$ denotes the norm of a vector field on $\Sigma$. Recall here that $a$ is a pastpointing null vector and $N$ is future-pointing, so that $\langle N, a\rangle>0$ on $\Sigma$.

In order to prove our main results, we will make use of the well-known Omori-Yau maximum principle [15], recalled next.

Lemma 2. Let $M$ be a complete Riemannian manifold whose Ricci curvature is bounded from below. If $u \in \mathcal{C}^{\infty}(M)$ is bounded from above on $M$, then there exists a sequence of points $\left\{p_{k}\right\}_{k \in \mathbb{N}}$ in $M$ such that

$$
\lim _{k \rightarrow \infty} u\left(p_{k}\right)=\sup _{M} u, \quad\left\|\nabla u\left(p_{k}\right)\right\|<1 / k \text { and } \Delta u\left(p_{k}\right)<1 / k .
$$

Now we are ready to state the following result.

Theorem 3. Let $\psi: \Sigma^{n} \rightarrow \mathcal{H}^{n+1}$ be a complete spacelike hypersurface with constant mean curvature $H$. If $\Sigma$ is bounded away from the infinity of $\mathcal{H}^{n+1}$, then $H=1$ necessarily. Moreover, in the 2-dimensional case, there exists $\tau$ such that $\Sigma^{2}=L_{\tau}$. 
Proof. The Gauss equation of $\Sigma$ in $\mathcal{H}^{n+1}$ describes the curvature of $\Sigma$, denoted by $R$, in terms of its shape operator, and it is given by

$$
\langle R(X, Y) X, Y\rangle=\langle X, X\rangle\langle Y, Y\rangle-\langle X, Y\rangle^{2}-\langle A X, X\rangle\langle A Y, Y\rangle+\langle A X, Y\rangle^{2}
$$

being $X, Y \in T \Sigma$. Taking traces here, we obtain that for every $X \in T \Sigma,\|X\|=1$,

$$
\begin{aligned}
\operatorname{Ric}(X, X) & =n-1+n H\langle A X, X\rangle+\langle A X, A X\rangle \\
& =n-1+\left\|A X+\frac{n H}{2} X\right\|^{2}-\frac{n^{2} H^{2}}{4} \geq n-1-\frac{n^{2} H^{2}}{4},
\end{aligned}
$$

where Ric stands for the Ricci curvature of $\Sigma$. Thus, the Ricci curvature of $\Sigma$ is bounded from below by the constant $n-1-n^{2} H^{2} / 4$. By assumption the function $u$ is bounded (from above and from below). Therefore, applying Lemma 2 to the function $u$, we know that there exists a sequence $\left\{p_{k}\right\}_{k \in \mathbb{N}}$ in $\Sigma$ such that

$$
\begin{gathered}
\lim _{k \rightarrow \infty} u\left(p_{k}\right)=\sup _{\Sigma} u<+\infty, \\
\left\|\nabla u\left(p_{k}\right)\right\|^{2}=\left\langle N\left(p_{k}\right), a\right\rangle^{2}-u^{2}\left(p_{k}\right)<1 / k^{2}
\end{gathered}
$$

and

$$
\Delta u\left(p_{k}\right)=n\left(H\left\langle N\left(p_{k}\right), a\right\rangle-u\left(p_{k}\right)\right)<1 / k .
$$

From the last equation, taking into account that $\langle N, a\rangle>0$ on $\Sigma$, we obtain that

$$
H<\frac{u\left(p_{k}\right)}{\left\langle N\left(p_{k}\right), a\right\rangle}+\frac{1}{n\left\langle N\left(p_{k}\right), a\right\rangle k}
$$

On the other hand, taking limits in (8) and using (7) we also get that

$$
\lim _{k \rightarrow \infty}\left\langle N\left(p_{k}\right), a\right\rangle=\lim _{k \rightarrow \infty} u\left(p_{k}\right)=\sup _{\Sigma} u .
$$

Therefore, taking limits in (9) we conclude that $H \leq 1$.

In an similar way, we may apply Lemma 2 to the function $-u$, obtaining another sequence $\left\{q_{k}\right\}_{k \in \mathbb{N}}$ in $\Sigma$ such that

$$
\begin{gathered}
\lim _{k \rightarrow \infty} u\left(q_{k}\right)=\inf _{\Sigma} u>0, \\
\left\|\nabla u\left(q_{k}\right)\right\|^{2}=\left\langle N\left(q_{k}\right), a\right\rangle^{2}-u^{2}\left(q_{k}\right)<1 / k^{2}
\end{gathered}
$$

and

$$
\Delta u\left(q_{k}\right)=n\left(H\left\langle N\left(q_{k}\right), a\right\rangle-u\left(q_{k}\right)\right)>-1 / k .
$$

Therefore, we now get that

$$
H>\frac{u\left(q_{k}\right)}{\left\langle N\left(q_{k}\right), a\right\rangle}-\frac{1}{n\left\langle N\left(q_{k}\right), a\right\rangle k},
$$

and taking limits again we conclude that $H \geq 1$. Summing up, $H=1$. Moreover, when $n=2$ we know by a result due to Akutagawa [2] and Ramanathan [13] that $\Sigma$ must be a totally umbilical surface of the foliation $L_{\tau}$. For the sake of completeness, we give here a different and direct proof of this assertion in our situation.

Hence, assume that $n=2$. Since $H=1$, (6) implies that the Gaussian curvature of $\Sigma$ is non-negative. Then $\Sigma$ is a complete Riemannian surface with non-negative Gaussian curvature, and by a classical result due to Ahlfors [1] and Blanc-FialaHuber [9], $\Sigma$ is parabolic, in the sense that any subharmonic function bounded from above on the surface must be constant. By (5) and from the fact that $\langle N, a\rangle>0$ we obtain that

$$
\langle N, a\rangle-u \geq 0
$$


on $\Sigma$. Then, (4) implies that $\Delta u \geq 0$. Therefore $u$ is a subharmonic function bounded from above on the parabolic surface $\Sigma$, and hence it must be constant, $\Sigma$ being one of the totally umbilical spacelike surfaces $L_{\tau}$.

Corollary 4. The only complete spacelike surfaces with constant mean curvature in $\mathcal{H}^{3}$ which are bounded away from the infinity are the totally umbilical surfaces $L_{\tau}$.

As a consequence of Lemma 1 and the proof of Theorem 3 we can also state the following result.

Theorem 5. Let $\psi: \Sigma^{n} \rightarrow \mathcal{H}^{n+1}$ be a complete spacelike hypersurface with constant mean curvature $H$. If $\Sigma$ is bounded away from the future infinity of $\mathcal{H}^{n+1}$ and has a future-pointing mean curvature vector, then $2 \sqrt{n-1} / n \leq H \leq 1$. Moreover, in the 2-dimensional case, there exists $\tau$ such that $\Sigma^{2}=L_{\tau}$.

Proof. In this case the function $u$ is bounded only from above. Therefore, by the first part of the proof of Theorem 3 we obtain that $H \leq 1$. On the other hand, estimate (6) implies that $H^{2} \geq 4(n-1) / n^{2}$. Otherwise by Bonnet-Myers' theorem we would get that $\Sigma$ is compact, which is not possible by Lemma 1. But the mean curvature vector field being future-pointing yields $H \geq 2 \sqrt{n-1} / n$. Summing up, $2 \sqrt{n-1} / n \leq H \leq 1$. When $n=2$ this gives $H=1$, and then $\Sigma$ must be one of the totally umbilical spacelike surfaces $L_{\tau}$.

Corollary 6. The only complete spacelike surfaces with constant mean curvature in $\mathcal{H}^{3}$ which are bounded away from the future infinity and have future-pointing mean curvature vector are the totally umbilical surfaces $L_{\tau}$.

It is worth pointing out that Corollary 6 is no longer true for surfaces which are bounded away from the past infinity of $\mathcal{H}^{3}$. Actually, in [11, Corollary 12] Montiel constructs complete spacelike hypersurfaces in $\mathcal{H}^{n+1}$ with constant mean curvature $H>1$ which are bounded away from the past infinity. On the other hand, in relation to our Corollaries 4 and 6, we also refer the reader to Theorem 4.4 and Theorem 4.5 in [5], where Caminha and de Lima have recently found other interesting characterizations of the totally umbilical surfaces $L_{\tau}$ in $\mathcal{H}^{3}$.

\section{An isometrically EQUiVAlENT MODEL. STEADY STATE TYPE SPACETIMES}

The steady state space can be expressed in an isometrically equivalent way as the generalized Robertson-Walker spacetime $-\mathbb{R} \times e^{t} \mathbb{R}^{n}$. That is, the real vector space $\mathbb{R}^{n+1}$ endowed with the Lorentzian metric tensor

$$
\langle,\rangle=-d t^{2}+e^{2 t}\left(d x_{1}^{2}+\ldots+d x_{n}^{2}\right),
$$

$\left(t, x_{1}, \ldots, x_{n}\right)$ being the canonical coordinates in $\mathbb{R}^{n+1}=\mathbb{R} \times \mathbb{R}^{n}$. To see it, take $b \in \mathbb{R}_{1}^{n+2}$, another null vector, such that $\langle a, b\rangle=1$ and let $\Phi: \mathcal{H}^{n+1} \rightarrow-\mathbb{R} \times e^{t} \mathbb{R}^{n}$ be the map given by

$$
\Phi(x)=\left(\log (\langle x, a\rangle), \frac{x-\langle x, a\rangle b-\langle x, b\rangle a}{\langle x, a\rangle}\right) .
$$

Then it can easily be checked that

$$
(d \Phi)_{x}(v)=\left(\frac{\langle v, a\rangle}{\langle x, a\rangle}, \frac{(v-\langle v, a\rangle b-\langle v, b\rangle a)\langle x, a\rangle-\langle v, a\rangle(x-\langle x, a\rangle b-\langle x, b\rangle a)}{\langle x, a\rangle^{2}}\right)
$$


and that $\Phi$ is an isometry between both spaces which conserves time orientation.

Therefore, a natural extension of the steady state space consists of considering the wider family of Lorentzian manifolds defined as follows. Let $M^{n}$ be a connected $n$-dimensional Riemannian manifold and consider the product manifold $\mathbb{R} \times M^{n}$ endowed with the Lorentzian metric tensor

$$
\langle,\rangle=-\pi_{\mathbb{R}}^{*}\left(d t^{2}\right)+e^{2 t} \pi_{M}^{*}\left(\langle,\rangle_{M}\right),
$$

where $\pi_{\mathbb{R}}$ and $\pi_{M}$ denote the projections from $\mathbb{R} \times M^{n}$ onto each factor and $\langle,\rangle_{M}$ is the Riemannian metric on $M$. For simplicity, we will write

$$
\langle,\rangle=-d t^{2}+e^{2 t}\langle,\rangle_{M}
$$

We will denote by $-\mathbb{R} \times e_{e^{t}} M^{n}$ the $(n+1)$-dimensional product manifold $\mathbb{R} \times M^{n}$ endowed with that Lorentzian metric, and we will refer to them as steady state type spacetimes. For instance, when $M^{n}$ is the flat $n$-torus we get the de Sitter cusp as defined in [6].

\section{SpaCelike hypersurfaces in A Steady STATE Type SPACEtime}

Let $\psi: \Sigma^{n} \rightarrow-\mathbb{R} \times_{e^{t}} M^{n}$ be a spacelike hypersurface. Observe that

$$
\partial_{t}=(\partial / \partial t)_{(t, x)}, t \in \mathbb{R}, x \in M,
$$

is a unitary timelike vector field globally defined on the ambient spacetime which determines a time-orientation on $-\mathbb{R} \times_{e^{t}} M^{n}$. Then, the future-pointing Gauss map of $\Sigma$ is the unique unitary timelike normal vector field $N$ globally defined on $\Sigma$ in the same time-orientation as $\partial_{t}$. Thus, we have

$$
\left\langle N, \partial_{t}\right\rangle \leq-1<0 \quad \text { on } \quad \Sigma .
$$

We will denote by $\Theta: \Sigma \rightarrow(-\infty,-1]$ the smooth function on $\Sigma$ given by $\Theta=\left\langle N, \partial_{t}\right\rangle$. Observe that the function $\Theta$ measures the hyperbolic angle $\theta$ between the futurepointing vector fields $N$ and $\partial_{t}$ along $\Sigma$. Indeed, they are related by $\cosh \theta=-\Theta$.

For a spacelike hypersurface $\psi: \Sigma^{n} \rightarrow-\mathbb{R} \times_{e^{t}} M^{n}$, we define the height function of $\Sigma$, denoted by $h$, as the projection of $\Sigma$ onto $\mathbb{R}$, that is, $h \in \mathcal{C}^{\infty}(\Sigma)$ is the smooth function given by $h=\pi_{\mathbb{R}} \circ \psi$. In the particular case when $M^{n}=\mathbb{R}^{n}$, by (10) the height function of $\Sigma$ can be written in terms of the function $u$ as

$$
h(p)=\log u\left(\Phi^{-1}(\psi(p))\right), \quad p \in \Sigma .
$$

Therefore, the umbilical hypersurfaces $L_{\tau}$, for which the function $u$ takes the constant value $u=\tau$, correspond to the slices $\{\log (\tau)\} \times \mathbb{R}^{n}$. Actually, for a general steady state type spacetime $-\mathbb{R} \times{ }_{e^{t}} M^{n}$, each leaf of the foliation $\{t\} \times M$ (called here a slice) of $-\mathbb{R} \times_{e^{t}} M^{n}$ is a totally umbilical spacelike hypersurface with constant mean curvature $H=1$.

According to the terminology introduced for hypersurfaces in the steady state space, we will say that a spacelike hypersurface $\Sigma$ in $-\mathbb{R} \times_{e^{t}} M^{n}$ is bounded away from the future infinity if there exists $\bar{t} \in \mathbb{R}$ such that

$$
\psi(\Sigma) \subset\left\{(t, x) \in-\mathbb{R} \times_{e^{t}} M^{n}: t \leq \bar{t}\right\},
$$

and we will say that $\Sigma$ is bounded away from the past infinity if there exists $\underline{t} \in \mathbb{R}$ such that

$$
\psi(\Sigma) \subset\left\{(t, x) \in-\mathbb{R} \times_{e^{t}} M^{n}: t \geq \underline{t}\right\}
$$


We will say that $\Sigma$ is bounded away from the infinity if it is bounded away from both the past and the future infinity. The following result is the natural extension of our Lemma 1

Lemma 7. Let $M^{n}$ be a Riemannian manifold. If $-\mathbb{R} \times_{e^{t}} M^{n}$ admits a complete spacelike hypersurface $\Sigma$ which is bounded away from the future infinity, then $M$ is necessarily complete and the projection $\Pi=\pi_{M} \circ \psi: \Sigma \rightarrow M$ is a covering map.

Proof. The proof follows the ideas of [3, Lemma 3.1]. Let $\psi: \Sigma^{n} \rightarrow-\mathbb{R} \times{ }_{e^{t}} M^{n}$ be a complete spacelike hypersurface and let $\Pi=\pi_{M} \circ \psi: \Sigma \rightarrow M$ denote its projection on $M$. Observe that

$$
\langle v, v\rangle=-\left\langle d \psi_{p}(v), \partial_{t}\right\rangle^{2}+e^{2 h(p)}\left\langle d \Pi_{p}(v), d \Pi_{p}(v)\right\rangle_{M} \leq e^{2 h(p)}\left\langle d \Pi_{p}(v), d \Pi_{p}(v)\right\rangle_{M}
$$

for every $p \in \Sigma$ and $v \in T_{p} \Sigma$. Thus, $\Pi^{*}\left(\langle,\rangle_{M}\right) \geq\left(1 / e^{2 h}\right)\langle$,$\rangle . As \Sigma$ is bounded away from the future infinity, it follows from here that

$$
\Pi^{*}\left(\langle,\rangle_{M}\right) \geq \frac{1}{e^{2 \bar{t}}}\langle,\rangle=\widetilde{\langle,\rangle}
$$

for a certain real number $\bar{t}$. Then reasoning as in the proof of Lemma 1, we get that $\Pi:(\Sigma, \widetilde{\langle,\rangle}) \rightarrow\left(M,\langle,\rangle_{M}\right)$ is a local diffeomorphism which increases the distance. The proof finishes by recalling that if a map from a connected complete Riemannian manifold $M_{1}$ into another connected Riemannian manifold $M_{2}$ of the same dimension increases the distance, then it is a covering map and $M_{2}$ is complete [10, Chapter VIII, Lemma 8.1].

Let $\psi: \Sigma^{n} \rightarrow-\mathbb{R} \times_{e^{t}} M^{n}$ be a spacelike hypersurface with height function $h$. Observe that the gradient of $\pi_{\mathbb{R}}$ on $-\mathbb{R} \times_{e^{t}} M^{n}$ is

$$
\bar{\nabla} \pi_{\mathbb{R}}=-\left\langle\bar{\nabla} \pi_{\mathbb{R}}, \partial_{t}\right\rangle \partial_{t}=-\partial_{t} .
$$

Therefore, the gradient of $h$ on $\Sigma$ is

$$
\nabla h=\left(\bar{\nabla} \pi_{\mathbb{R}}\right)^{\top}=-\partial_{t}^{\top},
$$

where $\partial_{t}^{\top}$ denotes the tangential component of $\partial_{t}$ along $\Sigma$, that is,

$$
\partial_{t}=\partial_{t}^{\top}-\Theta N \text {. }
$$

In particular,

$$
\|\nabla h\|^{2}=\Theta^{2}-1 .
$$

Let $Y$ denote the timelike vector field on $-\mathbb{R} \times e^{t} M^{n}$ given by

$$
Y(t, x)=e^{t}(\partial / \partial t)_{(t, x)}, \quad(t, x) \in-\mathbb{R} \times_{e^{t}} M^{n} .
$$

From the relation between $\bar{\nabla}$, the Levi-Civita connection of the ambient space $-\mathbb{R} \times e_{e^{t}} M^{n}$, and the Levi-Civita connection of $M$, we get that

$$
\bar{\nabla}_{Z} Y=e^{t} Z
$$

for any vector field $Z$ on $-\mathbb{R} \times_{e^{t}} M^{n}$. That is, $Y$ is a globally defined closed conformal vector field on $-\mathbb{R} \times_{e^{t}} M^{n}$. Writing $\partial_{t}=-\nabla h-\Theta N$ along the hypersurface $\Sigma$ and using Gauss and Weingarten formulae, it is not difficult to get from (12) that

$$
e^{h} X=\left\langle\nabla e^{h}, X\right\rangle \partial_{t}-e^{h} \nabla_{X} \nabla h+e^{h}\langle A X, \nabla h\rangle N+e^{h} \Theta A X-e^{h} X(\Theta) N
$$

for every $X \in T \Sigma$. Therefore,

$$
\nabla_{X} \nabla h=\Theta A X-X-\langle\nabla h, X\rangle \nabla h
$$


for every $X \in T \Sigma$, and the Laplacian on $\Sigma$ of the height function is given by

$$
\Delta h=-n H \Theta-\left(n+\|\nabla h\|^{2}\right) .
$$

Now we are ready to state the main result of this section, which is the natural extension of our Theorem 3 to the wider family of steady state type spacetimes.

Theorem 8. Let $M^{n}$ be a (necessarily complete) Riemannian manifold with nonnegative sectional curvature and let $\psi: \Sigma^{n} \rightarrow-\mathbb{R} \times_{e^{t}} M^{n}$ be a complete spacelike hypersurface with constant mean curvature $H$. If $\Sigma$ is bounded away from the infinity, then $H=1$. Moreover, when $n=2$, the surface $\Sigma$ is necessarily a slice $\{t\} \times M$.

Proof. The Gauss equation of $\Sigma$ is given by

$$
\langle R(X, Y) X, Y\rangle=\langle\bar{R}(X, Y) X, Y\rangle-\langle A X, X\rangle\langle A Y, Y\rangle+\langle A X, Y\rangle^{2}
$$

for every $X, Y \in T \Sigma$, where $R$ and $\bar{R}$ stand for the curvature tensor of $\Sigma$ and $-\mathbb{R} \times{ }_{e^{t}} M^{n}$, respectively. Taking traces here, we get that for every $X \in T \Sigma$, $\|X\|=1$,

$$
\begin{aligned}
\operatorname{Ric}(X, X) & =\sum_{i=1}^{m}\left\langle\bar{R}\left(X, E_{i}\right) X, E_{i}\right\rangle+n H\langle A X, X\rangle+\langle A X, A X\rangle \\
& \geq \sum_{i=1}^{m}\left\langle\bar{R}\left(X, E_{i}\right) X, E_{i}\right\rangle-\frac{n^{2} H^{2}}{4}
\end{aligned}
$$

where Ric stands for the Ricci curvature of $\Sigma$. On the other hand, taking into account the relationship between the curvature tensor of $-\mathbb{R} \times_{e^{t}} M^{n}$ and the curvature tensor $R_{M}$ of $M$ (as well as the derivatives of the warping function) [12, Proposition 42], we also have that

$$
\left\langle\bar{R}\left(X, E_{i}\right) X, E_{i}\right\rangle=e^{2 h}\left\langle R_{M}\left(X^{*}, E_{i}^{*}\right) X^{*}, E_{i}^{*}\right\rangle_{M}-\left\langle X, E_{i}\right\rangle^{2}+1,
$$

for $1 \leq i \leq n$, where $X^{*}=\left(\pi_{M}\right)_{*}(X)$ denotes the projection of the vector field $X \in T \Sigma$ onto $M$, that is, $X=X^{*}-\left\langle X, \partial_{t}\right\rangle \partial_{t}$. Denoting by $K_{M}\left(X^{*} \wedge E_{i}^{*}\right)$ the sectional curvature in $M$ of the 2-plane generated by $X^{*}$ and $E_{i}^{*}$, we have that

$$
\left\langle R_{M}\left(X^{*}, E_{i}^{*}\right) X^{*}, E_{i}^{*}\right\rangle_{M}=K_{M}\left(X^{*} \wedge E_{i}^{*}\right)\left\|X^{*} \wedge E_{i}^{*}\right\|_{M}^{2},
$$

where, as usual,

$$
\left\|X^{*} \wedge E_{i}^{*}\right\|_{M}^{2}=\left\langle X^{*}, X^{*}\right\rangle_{M}\left\langle E_{i}^{*}, E_{i}^{*}\right\rangle_{M}-\left\langle X^{*}, E_{i}^{*}\right\rangle_{M}^{2} .
$$

Using this in (15), we get from (14)

$$
\begin{aligned}
\operatorname{Ric}(X, X) & \geq e^{2 h} \sum_{i=1}^{n} K_{M}\left(X^{*} \wedge E_{i}^{*}\right)\left\|X^{*} \wedge E_{i}^{*}\right\|_{M}^{2}+n-1-\frac{n^{2} H^{2}}{4} \\
& \geq n-1-\frac{n^{2} H^{2}}{4} .
\end{aligned}
$$

Consequently, the assumption on the sectional curvature of $M$ implies that the Ricci curvature tensor of $\Sigma$ is bounded from below. The proof that $H=1$ now follows by applying Lemma 2 to the height function. Actually, since $h$ is bounded from above we know that there exists a sequence $\left\{p_{k}\right\}_{k \in \mathbb{N}}$ in $\Sigma$ such that

$$
\lim _{k \rightarrow \infty} h\left(p_{k}\right)=\sup _{\Sigma} h<+\infty, \quad\left\|\nabla h\left(p_{k}\right)\right\|^{2}=\Theta^{2}\left(p_{k}\right)-1<1 / k^{2}
$$


and

$$
\Delta h\left(p_{k}\right)=-n H \Theta\left(p_{k}\right)-\left(n+\left\|\nabla h\left(p_{k}\right)\right\|^{2}\right)<1 / k .
$$

Therefore $\lim _{k \rightarrow \infty} \Theta\left(p_{k}\right)=-1$, and taking limits in the last equation we get $H \leq 1$.

In a similar way, by applying Lemma 2 to the function $-h$ we obtain another sequence $\left\{q_{k}\right\}_{k \in \mathbb{N}}$ in $\Sigma$ such that

$$
\lim _{k \rightarrow \infty} h\left(q_{k}\right)=\inf _{\Sigma} h>-\infty, \quad\left\|\nabla h\left(q_{k}\right)\right\|^{2}=\Theta^{2}\left(q_{k}\right)-1<1 / k^{2}
$$

and

$$
\Delta h\left(q_{k}\right)=-n H \Theta\left(q_{k}\right)-\left(n+\left\|\nabla h\left(q_{k}\right)\right\|^{2}\right)>-1 / k .
$$

Thus $\lim _{k \rightarrow \infty} \Theta\left(q_{k}\right)=-1$ again, and taking limits in the last equation we get $H \geq 1$. As a consequence, $H=1$.

Consider now the 2-dimensional case. Since $H=1$, (16) implies that the Gaussian curvature of $\Sigma$ is non-negative and, therefore, $\Sigma$ is a parabolic surface. Moreover, (13), jointly with (11), implies that

$$
\Delta h=-2 \Theta-\left(2+\|\nabla h\|^{2}\right)=-2 \Theta-\left(1+\Theta^{2}\right)=-(\Theta+1)^{2} \leq 0 .
$$

Therefore, $h$ is a superharmonic function bounded from below on the parabolic surface $\Sigma$ and hence it must be constant.

\section{REFERENCES}

1. L. V. Ahlfors, Sur le type d'une surface de Riemann, C.R. Acad. Sci. Paris 201 (1935), 30-32.

2. K. Akutagawa, On spacelike hypersurfaces with constant mean curvature in the de Sitter space, Math. Z. 196 (1987), 13-19. MR907404(88h:53052)

3. L. J. Alías, A. Romero and M. Sánchez, Uniqueness of complete spacelike hypersurfaces of constant mean curvature in generalized Robertson-Walker spacetimes, Gen. Relativity Gravitation 27 (1995), 71-84. MR1310212 (95k:53073)

4. H. Bondi and T. Gold, On the generation of magnetism by fluid motion, Monthly Not. Roy. Astr. Soc. 110 (1950), 607-611. MR0044363 (13:4081)

5. A. Caminha and H. F. de Lima, Complete vertical graphs with constant mean curvature in semi-Riemannian warped products, http://arxiv.org/abs/math/0609602

6. G. J. Galloway, Cosmological spacetimes with $\Lambda>0$, Advances in Differential Geometry and General Relativity, 87-101, Contemp. Math. 359, Amer. Math. Soc., Providence, RI, 2004. MR2096155(2005m:53132)

7. S. W. Hawking and G. F. R. Ellis, The Large Scale Structure of Space-Time, Cambridge University Press, Cambridge, 1973. MR0424186 (54:12154)

8. F. Hoyle, A new model for the expanding universe, Monthly Not. Roy. Astr. Soc. 108 (1948), $372-382$.

9. A. Huber, On subharmonic functions and differential geometry in the large, Comment. Math. Helv. 32 (1957), 13-72. MR0094452 (20:970)

10. S. Kobayashi and K. Nomizu, Foundations of Differential Geometry, Vol. II, Interscience, New York, 1969. MR0238225 (38:6501)

11. S. Montiel, Complete non-compact spacelike hypersurfaces of constant mean curvature in de Sitter spaces, J. Math. Soc. Japan 55 (2003), 915-938. MR2003752 (2004h:53095)

12. B. O'Neill, Semi-Riemannian Geometry. With Applications to Relativity, Academic Press, New York, 1983. MR719023 (85f:53002)

13. J. Ramanathan, Complete spacelike hypersurfaces of constant mean curvature in de Sitter space, Indiana Univ. Math. J. 36 (1987), 349-359. MR891779 (88g:53067) 
14. S. Weinberg, Gravitation and Cosmology: Principles and Applications of the General Theory of Relativity, John Wiley \& Sons, New York, 1972.

15. S. T. Yau, Harmonic functions on complete Riemannian manifolds, Comm. Pure and Appl. Math. 28 (1975), 201-228. MR0431040 (55:4042)

Departamento de Matemáticas, Universidad de Murcia, E-30100 Espinardo, Murcia, SPAIN

E-mail address: albujer@um.es

Departamento de Matemáticas, Universidad de Murcia, E-30100 Espinardo, Murcia, SPAIN

E-mail address: ljalias@um.es 